\title{
Modelagem da Solução de integração Café por meio de Rede de Petri Coloridas
}

\author{
Luis Gustavo Tabile, Fabricia Roos-Frantz \\ ${ }^{1}$ Universidade Regional do Noroeste do Estado do Rio Grande do Sul (UNIJUÍ) \\ Departamento de Ciências Exatas e Engenharias \\ Rua Lulu Ilgenfritz, 480 - 98700-000 — Ijuí/RS -- Brasil \\ luis.tabile@sou.unijui.edu.br,frfrantz@unijui.edu.br
}

\begin{abstract}
Nowadays it is very common for companies to use a software ecosystem, these ecosystems can be made up of several applications, and it is increasingly necessary for these applications to be integrated with the intention that data and functionality can be shared between them, therefore, it is advisable to integrate applications because it brings a better cost benefit to the company that opts for this alternative. To this purpose, there are platforms that provide support for modeling and implementing integration solutions, one example of which is Guaraná. Before an integration solution can be implemented, it should be checked to avoid possible errors in the modeling, and thus prevent errors from being corrected only in late stages of its development. The purpose of this article is to model the integration solution known as Café using the Petri Nets formalism, thus generating a model that can serve as a basis for the verification and validation of this solution. We present a translation of the Café solution model, developed with the Guaraná DSL platform modeling language, to a model in Colored Petri Nets. It is hoped that this modeling can contribute to the process of verification and validation of integration solutions.
\end{abstract}

Resumo. Hoje em dia é muito comum o uso de um ecossistema de software por empresas, esses ecossistemas podem ser compostos por diversas aplicações, $e$ cotidianamente se vê mais necessário que essas aplicações sejam integradas com a intenção de que possam ser compartilhado dados e funcionalidades entre elas, sendo assim é aconselhável a integração de aplicações por trazer um melhor custo beneficio a empresa que opta por essa alternativa. Para esse fim, existem plataformas que fornecem suporte a modelagem e implementação de soluções de integração, um exemplo dessas plataformas é o Guaraná. Antes que uma solução de integração possa ser implementada, ela deveria ser verificada para evitar possíveis erros na modelagem, e assim evitar que erros sejam corrigidos apenas em etapas tardias do seu desenvolvimento. O objetivo deste artigo é modelar a solução de integração conhecida como Café utilizando o formalismo de Redes de Petri, gerando assim um modelo que pode servir como base para a verificação e validação desta solução. Apresentamos uma tradução do modelo da solução Café, desenvolvido com a linguagem de modelagem da plataforma Guaraná DSL, para um modelo em Redes de Petri Coloridas. Espera-se que esta modelagem possa contribuir com o processo de verificação e validação de soluções de integração. 


\section{Introdução}

Com o aumento do uso da tecnologia ao longo dos anos, aplicações de software foram desenvolvidas ou adquiridas de terceiros para dar suporte aos processos de negócios das empresas, resultando em um diversificado ecossistema de software. Neste contexto, mudanças nos processos de negócio existentes ou necessidade de inclusão de novos processos ocorrem constantemente, e o ideal é fazer uso das aplicações já existente no ecossistema de software para reutilizar dados e funcionalidades e dessa forma não ter que desenvolver novas aplicações a partir do zero. Em meio a essa demanda, as empresas buscavam soluções mais eficientes e com menor custo, o que deu origem ao termo integração de aplicações empresariais, do inglês Enterprise Application Integration (EAI) [Linthicum 2000] . A integração de aplicações consiste em integrar aplicações para que elas possam trocar dados e funcionalidades necessários para novos processos de negócio. Essas aplicações foram criadas geralmente sem a intenção de ser integradas, o que constitui um ecossistema de software heterogêneo e difícil de integrar, pois umas aplicações precisam ser adaptadas aos formatos e dados das outras aplicações, o que gera um custo adicional, muitas vezes alto.

A área de EAI proporciona um conjunto de metodologias, técnicas e ferramentas para a integração de aplicações de um ecossistema de software. Atualmente, existem diversas plataformas que dão suporte ao projeto, implementação, execução e monitoramento de soluções de integração [He and Xu 2014]. Geralmente, essas soluções possuem arquitetura pipes-and-filters e são baseadas nos padrões de integração documentados em [Hohpe and Woolf 2004]. Dentre as diversas plataformas de integração, estão: a OIC (ORACLE Integration Cloud) [Metin 2018], a plataforma Betalike-EAI ${ }^{1}$, e a Guaraná [Frantz et al. 2016], a qual oferece uma Domain Specific Language (DSL) para projetar soluções de integração de aplicações empresarias e um motor de execução.

A análise de uma solução de integração antes de sua implementação é um fator importante que deve ser considerado, pois melhora a qualidade da solução e reduz gastos com possíveis correções de erros, ainda na fase de projeto. Para essa análise deve ser utilizada uma ferramenta que seja capaz de modelar e representar formalmente os modelos conceituais desenvolvidos com a Guaraná DSL. Um formalismo bastante utilizado para a análise e simulação de sistemas discretos que envolvem processos estocásticos, que é o caso de um processo de integração, é o formalismo das Redes de Petri. Introduzidas por Carl Adam Petri [Petri 1966], elas oferecem uma representação gráfica, baseada em grafos, que facilita o processo de modelagem, além de possuir um formalismo e um conjunto de ferramentas que dão suporte à verificação, validação e simulação de sistemas [Molloy 1982, Jensen et al. 2007, Jensen and Kristensen 2009, van der Aalst and Stahl 2011].

O objetivo do trabalho é desenvolver um modelo em Rede de Petri Colorida para a solução Café, modelada em Guaraná DSL; o problema de integração Café é um exemplo clássico da literatura na área de EAI [Hohpe 2005]. Para a modelagem foi utilizada a ferramenta CPN, que pode ser acessada em: ${ }^{2}$, a qual permite a construção de um modelo de simulação para verificação e validação de uma solução de integração, na fase de projeto. A tradução do modelo Café para Redes de Petri co-

\footnotetext{
${ }^{1}$ http://www.betalike.com.br

${ }^{2} \mathrm{http} / / /$ cpntools.org/
} 
loridas foi baseada nos trabalhos de outros autores que já fizeram este tipo de modelagem [Fahland and Gierds 2013, Roos-Frantz et al. 2015].

O restante deste artigo está organizado da seguinte maneira: Na Seção 2, o modelo da solução Café é apresentado e o fluxo de execução da solução modelada na linguagem Guaraná DSL é explicado. Na Seção 3, é apresentado o modelo da solução com Redes de Petri e uma explicação sobre a linguagem e a plataforma que foi utilizada para modelar essa solução.

\section{Solução de Integração Café}

O modelo conceitual da solução de integração Café em Guaraná DSL é composto basicamente por portas (de entrada, de saída ou de solicitação/resposta), tarefas, slots e aplicações (veja Figura 1). O modelo representa como os pedidos são requisitados e processados em uma cafeteria. A comunicação entre os pedidos que chegam na solução e os pedidos que saem da solução é realizada por meio de portas que leem e portas que mandam mensagens. Depois que o caixa enviar o pedido à solução, ele não tem mais acesso a ele; o pedido vai para uma fila de mensagens, percorre o fluxo de execução de tarefas e é encaminhado para os baristas que preparam os pedidos e os devolvem a solução, por meio das portas de comunicação. Dessa forma, enquanto a mensagem do pedido flui pela solução, outros pedidos podem ser enviados, sem que haja a necessidade de espera.

O fluxo de execução da solução começa na porta de entrada P1, que tem a função de leitura dos pedidos, e após a leitura desses pedidos eles são encaminhados para a tarefa T1, que vai separar esses pedidos em diversas mensagens para cada tipo de bebida. Logo, a mensagem é encaminhada para uma tarefa T2, que tem função de despachar os pedidos, os quais podem ir para quaisquer um dos dois baristas: para o barista de bebidas quentes, caso seja uma bebida quente ou para o barista de bebidas frias, caso seja uma bebida fria. Em seguida, a tarefa T3 replica a mensagem para o barista, para que a aplicação solicite o preparo da bebida por meio das portas P2 e P3 e outra mensagem fica aguardando a resposta do barista sobre as informações do preparo da bebida. A tarefa T5 correlaciona a mensagem com as informações do pedido vindo do barista, com a mensagem que estava aguardando a reposta. Quando todas as bebidas de um pedido tiverem sido preparadas, elas são enviadas para o garçom por meio da porta P6, que realizará a entrega do pedido. O mesmo corre no fluxo de execução que comunica com as portas P4 e P5.

\section{Modelo da solução em Redes de Petri}

As Redes de Petri possuem dois elementos básicos: as transições (retângulos), que são responsáveis por executar as tarefas e os lugares (círculos), que são variáveis de estado [Desel and Reisig 2015]. As Redes de Petri são grafos bipartidos, nos quais os lugares sempre são direcionados para transições e transições para lugares. Lugares e transições são conectados por meio de arcos que são representados por flechas, as quais indicam o sentido e fluxo do token. Com o CPN Tools é possível modelar Redes de Petri Coloridas [Jensen et al. 2007], nas quais os tokens podem ser individualizados por meio de tipos diferentes, denominados cores.

No modelo da solução em Redes de Petri, ilustrado na Figura 2, foram utilizados 5 lugares que correspondem às portas de entrada P1, P3 e P5 e às portas de saída P2, P4 e P6. As transições "T"representam as tarefas a serem processadas, por exemplo, a tarefa 




Figura 1. Solução de integração Café [Frantz 2012]

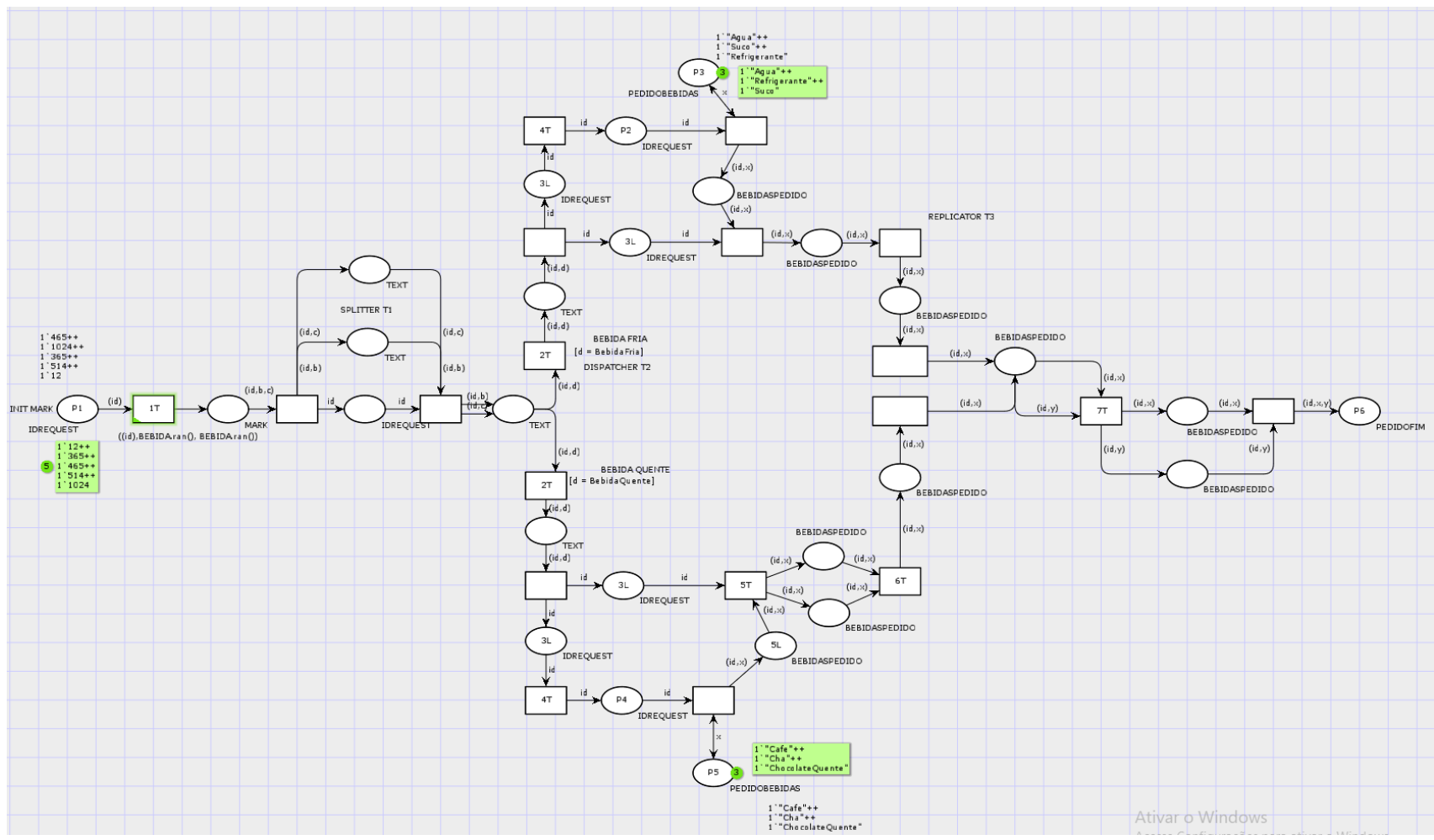

Figura 2. Representação da solução em Redes de Petri usando a ferramenta CPN Tools

T1 é responsável por dividir as mensagens em outras mensagens, que são direcionadas para as bebidas quentes e para as bebidas frias. A transição T1 representa a primeira tarefa, pronta para a execução, ou seja, ela está habilitada na marcação inicial porque possui tokens no lugar conectado ao seu arco de entrada. Quando T1 é disparada, ela 
consome esses tokens e produz outros tokens no lugar conectado ao seu arco de saída. Os lugares são representados pela letra "P"que representam variáveis de estado.

A solução começa na porta $\mathrm{P} 1$, que já contém 5 tokens prontos para a execução, nessa solução o cliente tem que fazer dois pedidos, não importa o tipo dos pedidos, e sim a quantidade deles, os pedidos podem ser dois frios, dois quentes ou um quente e um frio, cada token representa um número do pedido de um cliente. Esse pedido é encaminhado até a tarefa T1, a qual seleciona o tipo da bebida e em seguida é separa em dois tokens, que contêm o mesmo número do pedido, para que possam ser agrupados novamente no final da solução. Cada tarefa $2 \mathrm{~T}$ possui uma condição, para o pedido ir para o barista de bebidas frias o token tem que ser do tipo bebida fria, e para ir para o barista de bebidas quentes o token tem que ser do tipo bebida quente. Após ser orientado para o barista correto, a P3 é responsável pelo envio da bebida, é interessante observar que nessa P3 possui dois arcos direcionais, esses dois arcos funcionam de modo para que quando um pedido é feito o item que foi enviado para o cliente seja reabastecido.

Após a execução da aplicação, a porta $\mathrm{P} 6$ possui todos os pedidos que foram feitos (veja Figura 3). Ao total foram realizados 95 passos durante a execução. Pretende-se com essa modelagem criar uma solução com redes hierárquicas para ver o tempo que seria necessário para realizar esses 5 pedidos com 10 itens no total.

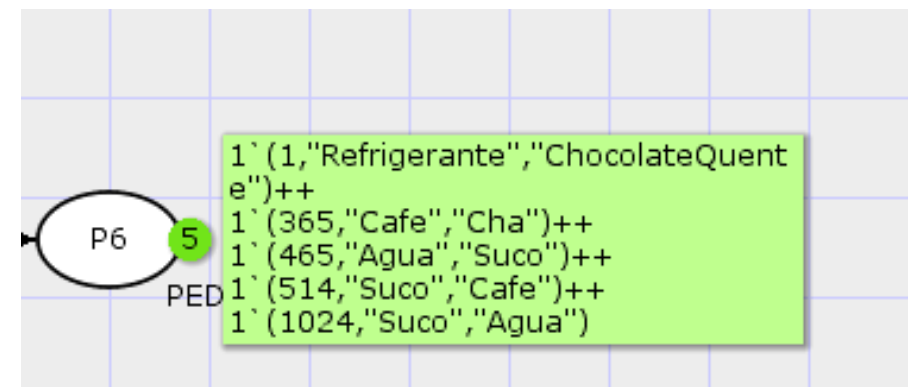

Figura 3. Resultado final após a execução da Solução de Integração Café

\section{Processos de Verificação do Modelo de Simulação}

Para a verificação do modelo de simulação apresentado nesse artigo foi utilizada uma técnica de verificação proposta por Sargent [Sargent 2005], a qual descreve o fluxo de mensagens para verificar se o modelo está implementado de forma correta. Esse modelo foi dividido em 3 blocos, separados por cores. O lugar P1 representa o inicio, que após 1000 passos realizados resultou em um total de 121 tokens disparados, a transição T5 e T10 possuem dois contadores de disparos, os demais numerais representam o número de mensagens que ficaram acumuladas ao final da execução.

A Figura 4, representa o diagrama de aplicação da técnica de Sargent, separada em 3 blocos de cores. As tarefas correlacionadoras (T8a, T8b) são alimentadas pelos slots S6a, S8a, S6b e S8b, em ambos lados, tanto na parte inferior quanto na superior é possível ver sinais de acúmulo de mensagens superior a média nesses slots, o que pode causar gargalos, pois as tarefas S8a e S8b dependem da execução da transição T9a e T9b, que dependem da execução das portas P2,P3,P4 e P5. O slot S4 acumulou cerca de 2 vezes mais mensagens que os demais, assim sendo, o slot que possui o maior acúmulo de mensagens, esse acúmulo acontece devido as condições que as tarefas T5a e T5b possuem, 
sendo a tarefa T5a possuindo uma condição caso a bebida seja Fria e T5b caso a bebida seja Quente.

A Figura 5 representa a aplicação da técnica de Sargent, é separada em 3 colunas, a primeira coluna é o bloco a qual a técnica foi realizada, a segunda coluna calcula o valor inicial de disparos da transição e compara realizando a soma do bloco, caso a soma seja a mesma do que o número de disparo da transição a coluna três dará um OK mostrando que naquele setor o modelo foi implementado de maneira correta. Após a analise da tabela chegamos ao resultado que todos os blocos atendem o principio da técnica de Sargent, pois o número de disparos da transição de entrada é igual a soma da fila com as saídas.

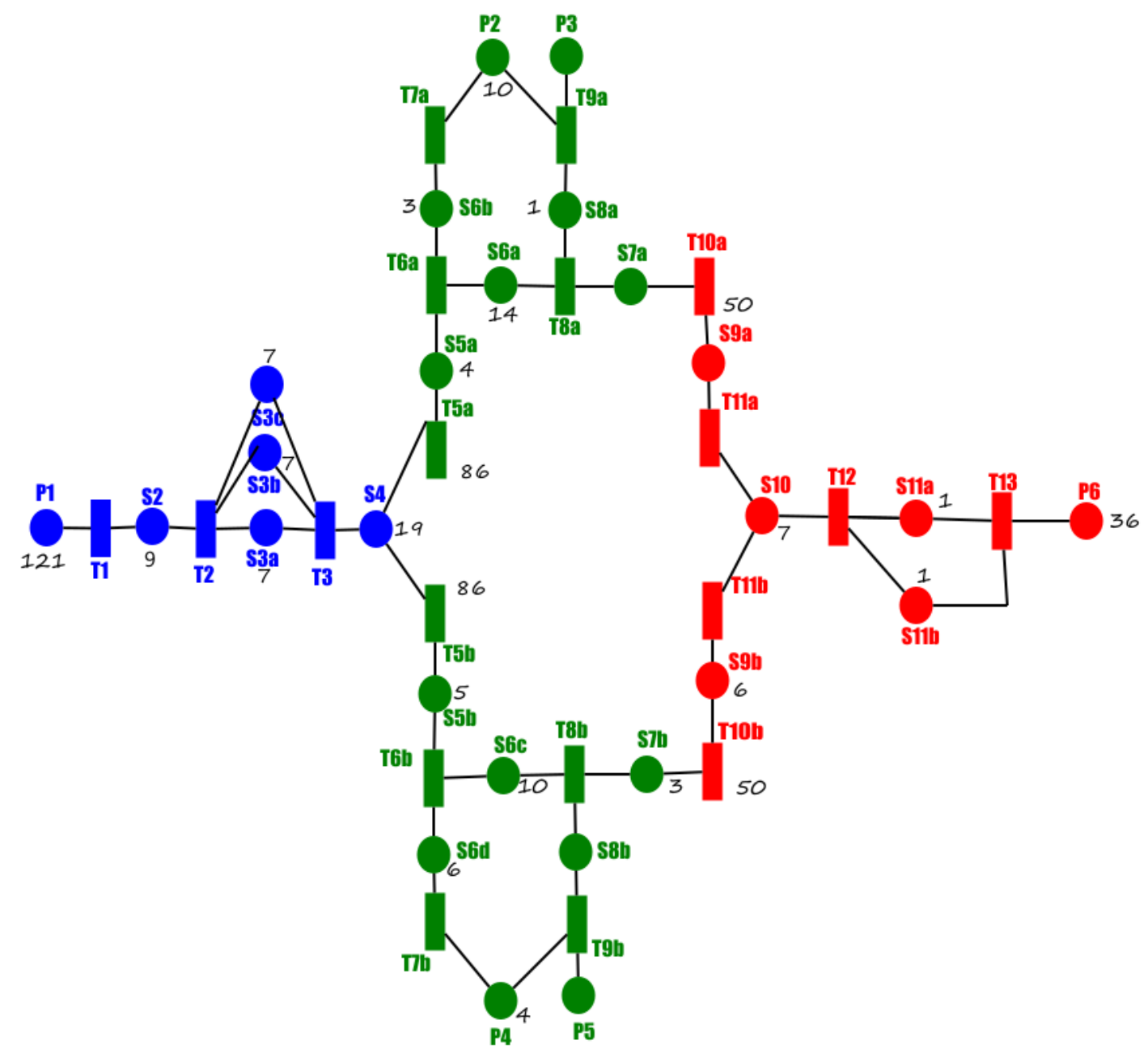

Figura 4. Diagrama de Aplicação da Técnica de Sargent

\section{Trabalhos Relacionados}

Na literatura pouco se é encontrado sobre uma solução de integração modelada em algum ambiente de simulação. Porém existem muitos trabalhos que tem alguma relação com Redes de Petri, pois é uma rede que abrange muitas áreas, como por exemplo, na modelagem e análise de processos de negócios. Durante a elaboração deste trabalho foram estudadas diversas tecnologias com objetivo parecidos ao que foi apresentado nesse artigo. 


\begin{tabular}{|c|c|c|}
\hline Bloco & Gontagem & Resultado \\
\hline Azul & $\mathrm{D}(\mathrm{P} 1)=\mathrm{D}(\mathrm{T} 1)+\mathrm{F}(\mathrm{S} 2)+\mathrm{D}(\mathrm{T} 2)+\mathrm{F}(\mathrm{S} 3 \mathrm{a})+\mathrm{D}(\mathrm{T} 3)+\mathrm{F}(\mathrm{S} 4)+\mathrm{D}(\mathrm{T} 5)$ & OK \\
\hline Azul & $121=9+7+19+86=121$ & OK \\
\hline Verde & $D(T 5)=F(S 5 a, b)+D(T 6 a, b)+F(S 6 a, b)+D(T 7 a, b)+P 2+P 4+D(T 8 a, b)+F(S 7 a, b)+F(S 8 a, b)+D(T 10)$ & OK \\
\hline Verde & $86=4+14+5+10+3+50=86$ & OK \\
\hline Vermelho & $D(T 10)=F(S 9 a, b)+D(T 11 a, b d)+F(S 10)+D(T 12)+F(S 11 a, b)+T 13+P 6$ & OK \\
\hline Vermelho & $50=6+7+1+36=50$ & OK \\
\hline
\end{tabular}

Figura 5. Aplicação da Técnica de Verificação .

O trabalho apresentado por Carl Adam Petri [Petri 1966] dá início a um modelo matemático, o qual chamamos hoje em dia de Rede de Petri, que é o alicerce dessa pesquisa. Hohpe [Hohpe and Woolf 2004] cita uma coleção de padrões de integração, 65 no total, usados para implementar um sistema de mensagens. A tradução feita por Fahland [Fahland and Gierds 2013] dos padrões de integração básicos descritos por [Hohpe and Woolf 2004] permitiu a criação da Modelagem da Solução de Integração Café por meio de Redes de Petri Coloridas apresentada nesse artigo.

O trabalho realizado por [Roos-Frantz et al. 2015] é o primeiro a propor o uso de Redes de Petri Estocásticas para analisar o comportamento de uma solução de integração feita com o Guaraná. Neste trabalho é proposto uma simulação usando Redes de Petri, para poder analisar soluções baseadas no Guaraná, o trabalho mostra que as Redes de Petri são uma ferramenta útil para simular soluções de integração. Também, demonstra que o modelo conceitual do Guaraná pode ser traduzido em um modelo em Redes de Petri estocásticas. Esse estudo é baseado em um problema de integração real, que tem como objetivo automatizar um processo de registro de novos usuários.

\section{Considerações Finais}

O desenvolvimento do presente estudo possibilitou uma representação de um modelo em Guaraná DSL para Redes de Petri Coloridas. Esse processo de modelagem servirá como suporte para a investigação e análise de modelos conceituais em Guaraná DSL para previsão de possíveis erros e gargalos, ainda na fase de projeto.

Acreditamos que este trabalho pode contribuir com um projeto mais completo sobre análise de processos de integração. O trabalho apresenta uma forma de transformar uma solução representada com Guaraná DSL em um modelo mais formal, para possibilitar verificação e simulação dessa solução em fase de projeto. O objetivo desta modelagem é contribuir com uma proposta de metodologia para a formalização da linguagem Guaraná DSL por meio de Redes de Petri

Ressaltamos que as maiores dificuldades encontradas na modelagem ocorreu frente as tarefas empregadas no modelo conceitual, e principalmente diante da criação de um método que possa aceitar mais pedidos ao mesmo tempo, que deve sempre ter um número fixo no caso desse modelo. 


\section{Agradecimentos}

Este trabalho tem o apoio da Fundação de Amparo à Pesquisa do Estado do Rio Grande do Sul (FAPERGS) no contexto do Programa Pesquisador Gaúcho, com termo de outorga número 17/2551-0001206-2 e da Universidade Regional do Noroeste do Estado do Rio Grande do Sul (UNIJUÍ) no contexto do Programa PIBIC/UNIJUÍ.

\section{Referências}

Desel, J. and Reisig, W. (2015). The concepts of petri nets. Software \& Systems Modeling, 14(2):669-683.

Fahland, D. and Gierds, C. (2013). Analyzing and completing middleware designs for enterprise integration using coloured petri nets. In Advanced Information Systems Engineering, pages 400-416. Springer Berlin Heidelberg.

Frantz, R. Z. (2012). Enterprise Application Integration - An Easy-to-Mantain ModelDriven Engineering Approach. $\mathrm{PhD}$ thesis, Universidad de Sevilla.

Frantz, R. Z., Corchuelo, R., and Roos-Frantz, F. (2016). On the design of a maintainable software development kit to implement integration solutions. Journal of Systems and Software, 111:89-104.

$\mathrm{He}, \mathrm{W}$. and Xu, L. D. (2014). Integration of distributed enterprise applications: A survey. IEEE Transactions on Industrial Informatics, 10(1):35-42.

Hohpe, G. (2005). Your coffee shop doesn't use two-phase commit. IEEE software, 22(2):64-66.

Hohpe, G. and Woolf, B. (2004). Enterprise integration patterns: Designing, building, and deploying messaging solutions. Addison-Wesley Professional, 1st edition.

Jensen, K. and Kristensen, L. M. (2009). Coloured Petri nets: modelling and validation of concurrent systems. Springer Science \& Business Media.

Jensen, K., Kristensen, L. M., and Wells, L. (2007). Coloured petri nets and cpn tools for modelling and validation of concurrent systems. International Journal on Software Tools for Technology Transfer, 9(3):213-254.

Linthicum, D. S. (2000). Enterprise application integration. Addison-Wesley Professional.

Metin, Y. (2018). Die nutzung von oracle integration cloud als ipaas-lösung für eine hybride integration. pages $48-67$.

Molloy, M. K. (1982). Performance analysis using stochastic petri nets. Computers, IEEE Transactions on, 100(9):913-917.

Petri, C. (1966). Kommunikation mit automaten. schriften des iim nr. 2, institut fur instrumentelle mathematic. Technical report, English translation: Technical Report RADCTR-65-377, Griffiths Air Base, New York.

Roos-Frantz, F., Binelo, M. O., Frantz, R. Z., Sawicki, S., and Basto-Fernandes, V. (2015). Using petri nets to enable the simulation of application integration solutions conceptual models. In International Conference on Enterprise Information Systems (ICEIS), pages 87-97. 
Sargent, R. G. (2005). Verification and validation of simulation models. Proceedings of the 37th conference on Winter simulation, pages 130-143.

van der Aalst, W. and Stahl, C. (2011). Modeling Business Processes: A Petri NetOriented Approach. The MIT Press. 